\title{
Integrating social determinants of health screening and referral during routine emergency department care: Evaluation of reach and implementation challenges
}

\section{Andrea S Wallace ( $\sim$ andrea.wallace@nurs.utah.edu )}

The University of Utah College of Nursing https://orcid.org/0000-0001-9694-2403

Brenda L. Luther

The University of Utah College of Nursing

Shawna M. Sisler

The University of Utah College of Nursing

\section{Bob Wong}

The University of Utah College of Nursing

Jia-Wen Guo

The University of Utah College of Nursing

\section{Research}

Keywords: social determinants of health, socioeconomic factors, emergency service, referral and consultation, health equity

Posted Date: March 31st, 2021

DOI: https://doi.org/10.21203/rs.3.rs-327585/v1

License: (9) (i) This work is licensed under a Creative Commons Attribution 4.0 International License. Read Full License 


\section{Abstract}

\section{Background}

Despite the importance of social determinants in health outcomes, little is known about best practices for screening and referral during clinical encounters. This study aimed to implement universal social needs screening and community service referrals in an academic emergency department (ED), evaluating for feasibility, reach, and stakeholder perspectives.

\section{Methods}

Between 01/2019-02/2020, ED registration staff screened patients for social needs using a 10-item, low literacy, English-Spanish screener on touchscreens that generated automatic referrals to a community service outreach specialists and data linkages. The RE-AIM framework guided evaluation. Reach was estimated through number of approaches, and completed screenings; effectiveness was estimated through receipt of community service referrals. Adoption was addressed qualitatively via content analysis and qualitative coding techniques from (1) meetings, clinical interactions, and semi-structured interviews with ED staff; and (2) an iterative "engagement studio" with an advisory group composed of ED patients representing diverse communities.

\section{Results}

Overall, 4608 participants were approached, and $61 \%$ completed the screener. The most common reason for non-completion was patient refusal (43\%). $47 \%$ of patients with completed screeners communicated one or more needs, $34 \%$ of whom agreed to follow-up by resource specialists. Of the 482 participants referred, $20 \%$ were reached by outreach specialists and referred to community agencies. Only $7 \%$ of patients completed the full process from screening to community service referral; older, male, non-white, and Hispanic patients were more likely to complete the referral process.

Iterative staff $(n=8)$ observations and interviews demonstrated that, despite instruction for universal screening, patient presentation (e.g., appearance, insurance status) drove screening decisions. Staff communicated discomfort with, and questioned the need for, screening. Patients $(n=10)$ communicated desire for improved understanding of their unmet needs, but had concerns about stigmatization and privacy, and communicated how receptivity of screenings and outreach are influenced by perceived sincerity of screening staff.

\section{Conclusions}

Despite limited time and technical barriers, few patients with social needs ultimately received service referrals. Perspectives of staff and patients suggest that social needs screening during clinical encounters should incorporate structure for facilitating patient-staff relatedness and competence, and address patient vulnerability by ensuring universal, private screenings with clear intent. 


\section{Contributions To The Literature}

- Despite limited evidence of technical or logistical barriers to universal social needs screening and referrals, few emergency department patients with social needs received community outreach.

- When screening for unmet social needs, health care systems need to overtly acknowledge the role of patient stigmatization based on presentation and insurance status.

- To decrease staff discomfort in asking questions about unmet social needs, interventions should be developed and tested to improve staff familiarity/comfort in delivering screening, improve understanding of how social needs have implications for care, and promote engagement between staff and patients.

\section{Background}

Over the last decade, it has become widely accepted that social determinants of health (SDOH), or the conditions in which people live, learn, work, and play (Centers for Disease Control and Prevention [CDC]; 2018) determine the vast majority of individual health outcomes (CDC, 2018). However, while addressing SDOH is seen as critical to improving health outcomes (Braveman et al., 2011), concerns about the ethical, privacy, practical implications of universal screening efforts have been raised (Garg et al., 2016), and there is scant research to help healthcare systems design and implement feasible and effective strategies for addressing SDOH.

Current research efforts have largely focused on evaluating the impact of SDOH interventions on health outcomes and costs. To date, most research in the area has been limited to program evaluations (Tsai et al., 2019; Walker \& Jackson, 2019), with a small number of randomized trials demonstrating that SDOH screening results in little improvement in traditional measures of physical health, or health system costs (Finkelstein et al., 2020). As a result, investigators continue to apply increasingly complex methodologies to examine SDOH, identify multi-level interventions for addressing SDOH, and seek measures that are both clinically meaningful to patients and sensitive to change. This work falls in contrast to healthcare systems rapidly adopting SDOH interventions to screen for more limited "social needs" (e.g., housing, food, transportation), which has resulted in increased discussion about the nature and content of SDOH screening instruments and in organizations serving as examples of implementation strategies (LaForge et al., 2018). However, one common theme of this work in research and practice settings is that it is underscored by the importance of developing and implementing social needs screening in a way that is linked to substantive and actionable processes and services to meet patient needs (Gottlieb et al., 2016; Sullivan, 2019).

One yet-to-be applied approach for examining SDOH interventions is to place them the larger landscape of behavior change interventions, the frameworks for which clearly outline that the first step in intervention effectiveness is participant engagement: interventions need to be delivered in a way that is 
well-received by patients and, through measurable steps, lead to having needs met. To date, research focusing specifically on patient engagement in SDOH-focused interventions have demonstrated that patients are generally receptive to social needs screening and referral processes when embedded within healthcare services, even in situations when needs cannot be met, and that patients understand the connection of how meeting social needs can improve their health (Hsu et al., 2019). However, others have found that, even when screening positive for social needs, a majority of patients may not wish to engage in interventions to have needs met. In a recent study, Tong (2018) studied 123 patients, $85 \%$ of whom screened positive for social needs, but only $3 \%$ wanted help with those needs. Similarly, in a preliminary study conducted by this research team (Wallace et al., 2020) 52\% with needs wished referral after screening. Collectively, these finding suggest that a key issue for research is to understand the limited reach of SDOH interventions, and whether and how social needs screening impacts therapeutic relationships (Hsu et al., 2019; Wallace et al., 2020).

While best practices for health related screening are broadly available (Heller, Parsons et al., 2020), and there are networks available for meeting social needs with community-based resources (United Way 211, 2020; AAFP, 2020), little is known about how to best develop and support patients' ability to complete referrals for needed services (Billioux et al., 2017). Further, there is little available research related to staff engagement in social needs interventions and whether and how staff may impact intervention delivery and patient receptivity. As such, the purpose of this study was to fully implement a universal screening for $\mathrm{SDOH}$, or social needs, in Emergency Department (ED) care using existing personnel and resources, evaluating for the reach and adoption of the intervention, and focusing particular attention on staff- and patient-perceptions acting as intervention barriers and facilitators.

\section{Social Needs Screening and Referral in Routine Emergency Department Care}

As the only place in the United States health care system where patients cannot be turned away for inability to pay, EDs care for a disproportionate number of low-income and uninsured patients (Venkatesh et al., 2019; Zhou et al., 2017). The work described in this paper capitalized on engagement between clinician-investigators and social service providers during which it was discovered that ED patients were commonly referred to the United Way's 2-1-1 (211) service by ED care management staff. The 211 service, part of a nationwide network supported by United Ways, provides a free-of-charge, comprehensive list of contact information for local resource providers who address common social needs (e.g., transportation, financial advice, food and housing assistance, etc.). The 211 service described in this work - Utah 211 is staffed 24 hours per day, 7 days per week by trained Information Specialists with access to an information pool of over 10,000 services in the Utah and surrounding states. Information Specialists, who are subject to routine quality oversight, and use HIPAA-compliant software to track service use, consumer demographics, reported needs, and consumer follow-up.

Our academic clinical-211 partnership applied an evidence-based social needs screener development addressed key aspects of the Psychometric and Pragmatic Evidence Rating Scale (PAPERS), including acceptability, relative advantage over existing methods, ease of completion, compatibility, organizational 
activities, informing clinical or organizational decision-making, cost, language accessibility, and assessor burden (training) (Lewis et al., 2018). A particular focus during screener development was also to identify needs subject to follow-up and service referrals by 211 after ED discharge, and engaged community and clinical stakeholders to develop, adopt, refine, and iteratively test the process using existing resources in the ED setting. The process used to develop and integrate the early iterations of the social needs screener addressed key pragmatic measures for implementation research. (Glasgow, 2013; Stanick et al., 2018, Wallace et al., 2020)

\section{Methods}

\section{Design Overview}

This study was guided by the RE-AIM framework to evaluate implementation components critical to understanding future population health impact (Klegas et al., 2005). A convergent mixed-methods design was applied, in which quantitative screening and qualitative staff- and patient-data were simultaneously collected, analyzed separately, and then merged to obtain a deeper understanding of the feasibility and acceptability of social needs screening and referrals (Creswell \& Plano Clark, 2011). All methods were reviewed and approved by the University of Utah's Institutional Review Board.

\section{Social Needs Screening and Referral Process}

This study was conducted with adult patients seen in the University of Utah University Hospital ED, a tertiary academic health sciences Level I trauma center. In January 2019, registration staff were trained in how to administer and document the 10-item English-Spanish social needs screener on touchpads and the Research Electronic Data Capture (REDCap; Harris et al., 2009, 2019) online system. Between January 2019 and April 2020, ED registration staff administered the screener as part of their standard workflow. Patients were allowed to either self- or verbally-respond to the questions. While asked to complete the screening with all patients, staff were asked to use their discretion to omit patients with cognitive impairment, trauma, language other than English, or residents of skilled nursing facilities.

Previous work established that, on average, patients are able to complete the 10 dichotomous yes-no social needs questions in less than 80 seconds (Wallace et al, 2020). Immediately following the screening questions, patients were asked to share their preferred contact information if they wished to be contacted by a Utah 211 information specialist within 48 hours of ED discharge. As part of our referral intervention, patients were given a written (via the touchscreen screener) and verbal (via registration staff) introduction to Utah 211 (211utah.org) describing it as a free service providing referrals to low- and no-cost community resources for needs, such as transportation, food, housing, and medications. All patients were asked if they would like referral regardless of indicating whether they had social needs.

Throughout the implementation trial, patient responses were tracked in REDCap. Real time contact and social need response information were shared with appointed 211 information specialists for all ED patients wishing outreach and service referrals. Patient zip codes were also shared to pre-emptively assist 
information specialists locate resources in patients' geographic area. Information specialists then proceeded to conduct outreach via telephone, text, and/or email, according to patient preferences. Information specialists entered encounter information regarding contacts and service referrals into their HIPAA complaint Mediaware database, linked via a unique identifier by which 211 encounter details were routinely imported into the REDCap database. Finally, patient demographic details were imported into REDCap from the UHealth Enterprise Data Warehouse.

\section{Stakeholder observations and interviews}

We gathered data about the adoption of the social needs screening through 1) individual observations and interviews with staff and other key stakeholders in the ED setting; and 2) a patient advisory group using a semi-structured interview guide in a community engagement laboratory setting.

Staff Perspectives. In iterative pilots (Wallace et al., 2020), staff communicated some resistance and skepticism whether the scope of their role should be expanded to incorporate the social needs screener. In order to better understand the context and perspective of staff, in-depth, iterative interviews and observations were conducted with 8 registration team members, representing approximately one-third of staff engaged in screening. Data were collected during clinical interactions and staff meetings by study investigators external to the department; demographic characteristics of staff members was not collected to ensure anonymity.

Interviews explored each individual's approach to the delivery of the screenings to their patients and families, as well as staff attitudes toward its perceived clinical utility, patient value, and overall acceptability (see Appendix $A$ for interview guide). All participants anonymously participated in these recorded interviews, which were later transcribed and analyzed. Memos from each interview were also recorded after each interaction and then synthesized at the end of each day as a group. Content analysis and qualitative coding techniques identified themes, and results were abductively analyzed through the lens relative to self-determination theory (see Appendices $B$ and $C$ ).

Patient Focus Group. A purposive sample of ED patients who had accessed care at a large urban tertiary $E D$ in the Intermountain West during the last 12 months $(n=10)$ was recruited through established hospital community advisory groups to participate in an iterative "community engagement studio." Community engagement studios are utilized in research as medium to dynamically interact with community stakeholders in order to receive feedback regarding the planning, design, implementation, and dissemination of interventions (Israel et al, 2019). Participants were prepared for the engagement studio via a telephone conversation during which the social needs screener and purpose of the intervention was shared. Participants were then asked to discuss the screening questions and intervention with the diverse communities they represented.

The engagement studio discussion, moderated by an experienced engagement team and observed by the research team, occurred in a single event over two hours to explore the strengths, weaknesses, opportunities, and challenges in our design, as well as concerns brought up in staff interviews (e.g. 
stigmatization, contextualization of the screener). The discussion was guided by the tenant that each participant in the group served as a representative of their community, and the group as a collective was meant to represent the diverse community populations of the greater patient population. This one-time focus group method was used to help highlight individual responses, as well a process to co-construct meaning as a group (Gubrium, 2012). As such, the research team focused on the substantive content shared, as well as the conversational dynamics (Morgan, 2010).

\section{Analysis}

Staff interviews and observations. Files and data were uploaded to Atlas.ti (Hwang, 2008) for qualitative analysis. To identify major themes and categories within the data, a detailed content and thematic analysis strategy was employed, leveraging a dual-coding system and verified audit trail. All data are electronically stored in a HIPAA-compliant cloud server.

From the initial substantive coding of each registration staff interview, the categorical properties iteratively emerged through fine-tuning code frequency and code comparison into broader themes. In parallel, results were abductively analyzed through the lens relative to self-determination theory. The role of self-determination theory was chosen via pilot data from a quantitative companion study, testing the feasibility of the intervention. It seeks to leverage the critical roles that competence, relatedness, and autonomy play in assisting patients or staff to become self-determined and engaged with the intervention (Deci \& Ryan, 1985). The text was analyzed for codes and categories to identify commonalities and differences in responses between participants as well as describing attitudinal or behavioral responses of participants (Appendix B).

Patient Focus Group. The focus group and individual responses were recorded, and transcribed verbatim, and iteratively coded using methods offered for descriptive thematic analysis (Kim et al., 2017; Sandelowski, 2000). In addition, analysis was guided by the Theory of Reasoned Action (TRA) to help discover individuals' motivation and beliefs related to completing social needs screening, participating in outreach and service connections. TRA assumes the predictors of a behavior are a person's actions and beliefs regarding a behavior and attitude (Glanz et al., 2008). As such, TRA components of attitudes, norms, and perceived control were examined to explain the intention and beliefs, and as such, predict behaviors of these participants.

\section{Results}

\section{Reach of Social Needs Screening and Referral Intervention}

Over 412 days (1/14/2019-2/29/2020), 4608 patients were approached (Fig. 1). 1660 (36\%) were not screened (refused $-43 \%$, too sick/trauma - 16\%). 2821 patients completed the screener. The average age of participants was 44.4 (17.8) years old (Table 1). The distribution of men and women was $45.0 \%$ vs. $55.0 \%$, respectively. Of those completing screening, $14.2 \%$ were identified in their health record as Hispanic/Latino ethnicity, and 79\% were listed as White/Caucasian for racial background. 
Table 1

Participants Expressing Social Needs vs. No Social Needs

\begin{tabular}{|c|c|c|c|c|}
\hline & No needs & $\begin{array}{l}\text { One or more } \\
\text { needs }\end{array}$ & Total & \\
\hline Characteristic & $N=1497$ & $N=1324$ & $N=2821$ & $\begin{array}{l}p- \\
\text { value }\end{array}$ \\
\hline Age in years (SD) & $47.1(19.4)$ & $41.2(15.2)$ & $44.4(17.8)$ & $<0.001$ \\
\hline Gender & & & & 0.136 \\
\hline Female & $820(56.4 \%)$ & $679(53.5 \%)$ & $\begin{array}{l}1499 \\
(55.0 \%)\end{array}$ & \\
\hline Male & $635(43.6 \%)$ & $590(46.5 \%)$ & $\begin{array}{l}1225 \\
(45.0 \%)\end{array}$ & \\
\hline Ethnic Background & & & & $<0.001$ \\
\hline Chose not to disclose & $12(0.8 \%)$ & $8(0.6 \%)$ & $20(0.7 \%)$ & \\
\hline Hispanic/Latino & $158(10.9 \%)$ & $229(18.1 \%)$ & $387(14.2 \%)$ & \\
\hline Not Hispanic/Latino & $\begin{array}{l}1283 \\
(88.3 \%)\end{array}$ & $1030(81.3 \%)$ & $\begin{array}{l}2313 \\
(85.0 \%)\end{array}$ & \\
\hline Race & & & & $<0.001$ \\
\hline American Indian and Alaska Native & $9(0.6 \%)$ & $20(1.6 \%)$ & $29(1.1 \%)$ & \\
\hline Asian & $41(2.8 \%)$ & $11(0.9 \%)$ & $52(1.9 \%)$ & \\
\hline Black or African American & $35(2.4 \%)$ & $72(5.7 \%)$ & $107(3.9 \%)$ & \\
\hline Choose not to disclose & $5(0.3 \%)$ & $7(0.6 \%)$ & $12(0.4 \%)$ & \\
\hline $\begin{array}{l}\text { Native Hawaiian and Pacific } \\
\text { Islander }\end{array}$ & $25(1.7 \%)$ & $19(1.5 \%)$ & $44(1.6 \%)$ & \\
\hline Other & $135(9.3 \%)$ & $198(15.6 \%)$ & $333(12.2 \%)$ & \\
\hline White or Caucasian & $\begin{array}{l}1203 \\
(82.8 \%)\end{array}$ & $940(74.2 \%)$ & $\begin{array}{l}2143 \\
(78.8 \%)\end{array}$ & \\
\hline
\end{tabular}

Of the 2821 participants screened (Fig. 1), 1324 (46.9\%) indicated having one or more social needs. Of the 1324 participants indicating having one or more social needs, $453(34.2 \%)$ wished referral to 211 . In addition, there were 29 participants that asked to be referred to 211 who indicated having no social needs. Of the 482 participants who were referred to 211, 98 (20.3\%) were eventually reached by 211 
information specialists and were given referrals to community-based agencies. The overall percentage of those with needs who were given referrals to community-based agencies was $7.4 \%(98 / 1324)$ or $3.5 \%$ of the total sample screened $(98 / 2821)$.

In an effort to understand demographic factors contributing differences in reported social needs, we compared demographic characteristics (age, gender, race, and ethnicity) between the 1324 patients who indicated one or more needs vs. the 1497 patients who indicated no needs. Results (Table 1) show the patients who indicated one or more needs were significantly younger with 41.2 (15.2) years for those with needs vs. 47.1 (19.4) years without needs, $p<0.001$. There was a higher percentage of Hispanic ethnicity (18.1\% vs. $10.9 \%), p<0.001$, and a higher percentage of Black or African American (5.7\% vs. $2.4 \%$ ) and Other racial backgrounds ( $15.6 \%$ vs. $9.3 \%), p<0.001$ for those with one or more social need. There were no gender differences, $p=0.136$.

Next, to better understand intervention engagement, we compared demographic characteristics between the 98 participants who received community service referrals vs. the 384 participants who asked for outreach but did not connect with the 211 information specialist for community service referrals. Results (Table 2) indicate that patients who received referrals were older (46.5 (17.0) years vs. 42.3 (14.2) years, $p=0.029)$. Those who received service referrals were more likely to be of Hispanic ethnicity $(32.3 \% \mathrm{vs}$. $20.6 \%), p=0.042$; have a non-White racial background ( $32.3 \%$ vs. $16.9 \%), p=0.004$; or be male $(62.8 \%$ vs. $47.5 \%), p=0.008$. The average number of needs reported was not significantly different, with $4.7(2.5)$ needs reported in the group that received service referrals vs. 4.8 (2.8) needs in the group that did not receive service referrals, $p=0.666$. 
Table 2

Comparison between Receivers vs. Non-Receivers of 2-1-1 Referrals

\begin{tabular}{|llll|}
\hline & No 2-1-1 Referrals & Received 2-1-1 Referrals & \\
\hline Characteristic & $\mathrm{N}=384$ & $\mathrm{~N}=98$ & p - value \\
\hline Number of needs reported (SD) & $4.8(2.8)$ & $4.7(2.5)$ & 0.666 \\
\hline Age in years (SD) & $42.3(14.2)$ & $46.5(17.0)$ & 0.029 \\
\hline Gender & & & 0.008 \\
\hline Female & $189(52.5 \%)$ & $35(37.2 \%)$ & \\
\hline Male & $171(47.5 \%)$ & $59(62.8 \%)$ & \\
\hline Ethnic Background & & & 0.042 \\
\hline Choose not to disclose & $3(0.8 \%)$ & $0(0.0 \%)$ & \\
\hline Hispanic/Latino & $74(20.6 \%)$ & $30(32.3 \%)$ & \\
\hline Not Hispanic/Latino & $283(78.6 \%)$ & $63(67.7 \%)$ & \\
\hline Race & & & \\
\hline American Indian and Alaska Native & $4(1.1 \%)$ & $2(2.2 \%)$ & \\
\hline Asian & $2(0.6 \%)$ & $2(2.2 \%)$ & \\
\hline Black or African American & $24(6.7 \%)$ & $8(8.6 \%)$ & \\
\hline Choose not to disclose & $3(0.8 \%)$ & $0(0.0 \%)$ & \\
\hline Native Hawaiian and Pacific Islander & $10(2.8 \%)$ & $0(0.0 \%)$ & \\
\hline Other & $61(16.9 \%)$ & $30(32.3 \%)$ & \\
\hline White or Caucasian & $256(71.1 \%)$ & $51(54.8 \%)$ & \\
\hline * Overall missing demographics data $=5.9 \%$ & & \\
\hline
\end{tabular}

Of the 1324 patients who indicated a social need, utilities were the most requested social need (668, $50.5 \%)$, followed by rent/mortgage $(663,50.1 \%)$, and clothing/furniture $(655,49.5 \%)$. Please see Table 3 for other social needs. The most common referral provided by 211 was for utility services assistance (29 participants, $29.6 \%$ ), followed by rent payment assistance (26 participants, $26.5 \%$ ), and food pantries (24 participants, $24.5 \%$ ). Please see Table 4 for the six most common referrals provided. 
Table 3

Frequency of Social Needs $(\mathrm{N}=1324)$

\begin{tabular}{|llll|}
\hline Social Need & Yes & No & Prefer not to answer \\
\hline Utilities & $668(50.5 \%)$ & $632(47.7 \%)$ & $24(1.8 \%)$ \\
\hline Rent/Mortgage & $663(50.1 \%)$ & $638(48.2 \%)$ & $23(1.7 \%)$ \\
\hline Clothing/Furniture & $655(49.5 \%)$ & $647(48.9 \%)$ & $22(1.7 \%)$ \\
\hline Doctor/Medical visit & $605(45.7 \%)$ & $707(53.4 \%)$ & $12(0.9 \%)$ \\
\hline Food & $594(44.9 \%)$ & $710(53.6 \%)$ & $20(1.5 \%)$ \\
\hline Employment & $540(40.8 \%)$ & $761(57.5 \%)$ & $23(1.7 \%)$ \\
\hline Medication & $486(36.7 \%)$ & $818(61.8 \%)$ & $20(1.5 \%)$ \\
\hline Housing & $422(31.9 \%)$ & $888(67.1 \%)$ & $14(1.1 \%)$ \\
\hline Transportation & $309(23.3 \%)$ & $1005(75.9 \%)$ & $10(0.8 \%)$ \\
\hline Childcare/Eldercare & $191(14.4 \%)$ & $1107(83.6 \%)$ & $26(2.0 \%)$ \\
\hline
\end{tabular}

Table 4

Top Six Referral Types

\begin{tabular}{|ll|}
\hline Referral Type & $\mathbf{N}=98$ \\
\hline Utility Service Payment Assistance & $29(29.6 \%)$ \\
\hline Rent Payment Assistance & $26(26.5 \%)$ \\
\hline Food Pantries & $24(24.5 \%)$ \\
\hline Low Income/Subsidized Rental Housing & $16(16.3 \%)$ \\
\hline Navigator Programs & $8(8.2 \%)$ \\
\hline Food Stamps/SNAP Applications & $8(8.2 \%)$ \\
\hline
\end{tabular}

\section{Adoption}

\section{Staff Interviews and Observations}

Themes from registration staff included 1) using professional intuition to decide whether, when, and who to screen; 2) determining the appropriateness of staff being the "right" group to own this workflow; and 3) determining if the chosen screener is the "right" tool for the ED system. There is also strong evidence to 
supporting our hypothesis from our early pilot work that motivation (self-determination) plays a central role in adoption practices among front-line staff (Wallace et al., 2020). Staff who communicated that they felt like important members of the healthcare team, agents of change, or their role served a bigger purpose in the lives of patients - or intrinsic motivation - were more likely to integrate social needs screenings. In contrast, those communicating extrinsic motivation - feeling role expectations were prescribed by others - were likely to question their role in screening or to be skeptical of referrals to outside community resource agencies.

The vast majority of registration staff reported leveraging their own "professional intuition" to deliver the social needs screen in what they saw was the more effective way. Such augmentations occurred during the introduction of the screen (the framing) or during the decision to screen (the value). Assumptions for these personal algorithms were based primarily on the staff's view of the patient needs, specifically regarding insurance coverage, patient characteristics or demographics, current diagnoses, and/or a patient's ability to engage with the screener. All such heuristics were noted as staff "judgment calls" occurring within moments of meeting the patient.

Observed approaches to screening ran the gamut, from completely confident to unsure, skeptical, and resentful. The staff felt strongly that, given their role and experience, that their professional intuition was fine-tuned enough to understand which patients may be benefit (or not benefit) from the resultant services and to which the staff would alter their approach. About half of the participants also admitted to using their professional intuition to modify and/or take creative liberties "to make the screen [their] own." Meaning, the staff would try to maintain each items' integrity, but try to find more creative ways to ask or address the sensitive question line, etc. Staff with higher self-reported professional intuition and intrinsic motivation utilized more modifications and took more creative liberty in delivering the screener. These phenomena may be a result of the staff leveraging (and taking pride) in their cultivated experience or connection with patients. In terms of the professional role, there was little disagreement regarding the importance of screening for unmet social needs and its impact on the health of patients. One participant noted that, "It is more than the patient, it is about the staff trusting that the information is useful."

\section{Patient-Community Advisory Group}

Community participants were mostly $(n=8,80 \%)$ women, and ranged in age from $25-60$ years. The racial-ethnic composition of the group was $40 \%$ White, $20 \%$ Black, $10 \%$ Asian/Pacific Islander; $20 \%$ of the group identified as Hispanic/Latino. Educational preparation included $10 \%$ high school graduates, $40 \%$ some college, and $20 \%$ college graduates. Participants' annual household Incomes ranged from \$10,000 to $\$ 75,000$ or more, and number of members in participants' households ranged from 2-5

Utilizing the "engagement studio" format, feedback revealed an important evolution of perspectives regarding social needs screening. Initially, there was a consensus on the value of the mission; each individual patient-participant spoke of the importance in health care systems addressing a community's unmet social needs. When exploring the implementation of screening, there was critical feedback about the context and communication of the intervention emerged. The major themes - potential 
embarrassment, need for sincerity, and mitigating vulnerability - highlighted the important nuance when delivering social needs screening, and concerns about possible impact on intervention adoption.

Theme 1: Potential embarrassment, "Would I answer? Yes, Maybe, No"

Participant responses to the social needs screener were generally positive, with quotes of: 'for me, I won't have any problem', 'I'd be fine', and simply 'I've done these before'. The tone of initial conversations was casual, in which most participants confidently communicated that they would have no problem answering the social needs screening questions. Neutral comments were about length of screening, if they would be feeling good enough to answer, or if language barriers might play into decisions regarding whether or not to respond to screening questions.

Yet, quickly, the participants moved their responses from themselves to thoughts of 'others' and what others in their communities would do when asked to fill out the social needs screening. When they discussed what others would do, the responses transitioned to neutral (maybe they would do) as well as to negative (no, they would not do). As the participants continued to respond through the view of how 'others' may feel, they identified negative comments noting that people could be embarrassed, or that the screening could be seen as an invasion of personal privacy. The conversation explored more negative comments, divided out and developed into the second theme of relatedness and vulnerability.

Theme 2: Need for sincerity - "I need to know YOU before I answer"

After participants agreed they themselves would answer screening questions, they were asked 'who' should ask these questions. The theme of 'I need to know YOU before I answer' developed from responses. The need for trust, comradery, and familiarity were described as participants stated they would disclose the personal information of social needs to those they felt 'were genuine', 'were around the most', and 'who showed they cared'.

Questions related to how 'showing you care' looks to these participants were offered as a probe, to which participants answered 'eye contact', 'good personality', 'I liked her', 'you can just tell they care' were offered. One participant commented not 'showing you care' is not expected of some staff such as registration staff offering 'I know they are here about the business, the money'.

An important discovery in this sub-theme was that, for participants to feel comfortable answering, they need/want to know the person who is asking the social needs screening questions, or feel a connection to them through a caring attitude, or a role with the expectation of caring for them. Specific examples offered by participants were nurses or social workers.

Theme 3: Vulnerability - "These questions make me vulnerable"

Vulnerability arose as a theme from these patient-participants as they explored how they would feel when asked about their own social needs. Participants identified the main areas from which feelings of vulnerability stemmed, including: 1) a patient who has needs may imply they are unable to provide for themselves; 2) referring for needs increases a patients' exposure to outside agencies; and 3) they would 
not want these needs documented in the records that would "follow them around" in all clinical interactions. Participants reported particular community groups to which they would avoid disclosing their social needs due, in part, to their culture or past negative experiences applying for services; participants expressed concern that disclosing needs could increase a person's vulnerability and open their exposure to systems outside of healthcare. One participant offered the example of revealing that childcare is a challenge may inadvertently expose a patient to action by child protective services. When prompted by the question about how screening results might be communicated to their health care providers, participants unanimously stated they would not want screening results in their medical record for fear that doing so would lead to being treated "differently" by health care providers. Further, they communicated concern that information may follow patients after personal circumstances change.

\section{Discussion}

This study examined the reach and adoption of a HIT-facilitated social needs screening and referral intervention; each arm represented a key aspect in the success of its design and implementation. Despite sparse evidence of technical and logistical barriers to universal social needs screening in the ED, fewer than $64 \%$ of targeted patients were screened, and $7 \%$ of those who communicated one or more social need ultimately connected to services to address stated needs. Themes that emerged through qualitative data uncovered key, concrete messaging and training that may impact reach, adoption and, ultimately, effectiveness of SDOH-social needs screening and referral efforts in clinical settings.

Our experiences of suboptimal intervention reach are not unique in a landscape of clinical interventions attempting to address patients' social needs. A recent study by Hsu and colleagues (2019) found that fewer than half of their SDOH intervention participants reported resolution of social needs. These findings, combined with our own, suggest that barriers to SDOH intervention effectiveness likely exist both upstream and downstream from the point of service provider connections. While our own intervention was developed with clinical implementation in mind (i.e. ease of delivery and existing staff and resources), our analysis of intervention reach clearly illustrates the impact of staff and patient level factors act at multiple levels of the intervention, from decisions to approach, to complete screening, to accept outreach and, while not measured in this study, to ultimately act on referrals. Also, similar to Hsu and colleagues' (2019) conclusion that patient collaboration, empathy, and positive regard is a product of interventionists acting as advocates, patients in our study communicated the need for relationship building and other signs the connote sincerity on behalf of those administering the intervention.

Overall, our study results may highlight the need to firmly place SDOH interventions into the context of health behavior interventions, as influenced by concepts such as self-determination. Even in cases where a patient declines service referral, the work suggests benefits to the screeners' inclusion in clinic workflow; the assessment still informs staff-provider-patient interactions through a change in prescribed care and general knowledge regarding patient status. The fact that patients universally saw the impact of social needs and wanted their needs communicated to caring individuals suggest that, even when declining help in addressing social need, screening may still inform clinician-patient interactions. As reported by Tong et 
al (2018), despite reporting that assessing social needs is difficult and resource-intensive, clinicians also reported that knowing the patient had a social need changed care delivery and helped improve interactions with, and knowledge of, patients.

Complementary to the input of patients, our interview and observational data with staff demonstrate the need for additional, structured scripting and training related to presenting the screen's question delivery, both in terms of overcoming time barriers but also in navigating staff-patient discomfort. Interventions to facilitate connectedness may be also be useful, from the time of screening to ongoing engagement and problem solving for those open to referrals. To overcome discomfort and stigma of screenings, health systems seeking to connect screens and community referrals need to carefully consider strategies to better identify and promote staff's intrinsic motivation and facilitate implementation readiness on the part of individual staff. Patient responses demonstrated how skills of communicating trust and commitment to building trust are a critical prerequisite for staff who are asking about patients' unmet social needs. However, while allowing involved staff to pick and choose whether or how to implement the screen may facilitate staff autonomy, too much customization (i.e. leaving it up to staff to skip or modify the assessment) may threaten the fidelity of the screen. As such, our findings suggest that developing clear methods for addressing these areas related to the staff experience of carrying out social needs screening, and evaluating their impact on reach and effectiveness, may be a fruitful area for future research.

Facilitating intrinsic motivation and readiness could be further aided by more directive policies. We repeatedly found value in leveraging staff intuition about how/when to screen; they are most aware of the workflow's opportunities and challenges to integration. To maximize adoption of any intervention, it is critical to integrate the priorities and context of key stakeholders, as well as maximize existing system infrastructure. This study argues that by formally recognizing these "human factors" of screening, we can better frame interventions to increase adoption and feasibility across clinical practice and roles. Finally, by understanding staff underlying motivation and autonomy, complex human variables, such as staff discomfort and stigma of social needs screening, can be operationalized and challenged through targeted education and workflow, thereby increasing relatedness and decreasing resistance.

One unambiguous and strong concern common to both patients and staff participants was related to documenting social needs in the electronic health records. More specifically, patients voiced strong, unambiguous concerns over permanently documenting what they hope would are temporary life situations. This has important implications for efforts seeking to integrate SDOH-social needs data into clinical information systems: investments to insert and exchange these data in electronic health records are underway (Cantor \& Thorpe, 2018; Gottlieb et al., 2016). While clinicians may view social needs information as an important part of personalized care, patients also see this is different - and potentially lesser - care via patient profiling. Patients viewed screening only certain patient populations (e.g. by Medicare insurance) very unfavorably, and such efforts are even likely to undermine therapeutic relationships. 
While reach was limited overall, our quantitative data are somewhat reassuring in that those ultimately engaging in the 211 outreach and receiving referrals are those who are more often members of underserved and underrepresented patient populations. However, our experiences demonstrate that screening for social needs in a busy ED setting, or likely any health care setting, may be difficult to maintain as a sustained and accurate practice without staff mechanisms for ongoing staff engagement and feedback mechanisms. Health systems seeking to address social determinants by integrating social needs assessments and community-based referrals must carefully consider policies that prepare staff by identifying and promoting their intrinsic motivation and facilitating readiness, and on methods for communicating sincerity and concern for patients' individual circumstances. Additionally, because of the variability of resources and workflows across clinical settings, research should pursue a better understanding of what and where there is flexibility within the protocol for the setting and staff to customize their approach.

\section{Conclusions}

In contrast to the time and technology barriers commonly reported with SDOH screens in busy clinical settings, our study demonstrates that the reach and effectiveness of interventions aiming to address patients' social needs are strongly influenced key staff- and patient-factors related to stigma, privacy, and relatedness. Staff and patient perceptions of screenings suggest that, before scaling, implementation efforts ought to be accompanied by language that clearly communicates the universal nature and benefit of screening. Additionally, addressing the key pillars of self-determination theory - autonomy, competence, and relatedness - may serve as a useful framework for SDOH screening interventions. Future research and health system interventions should aim to test methods for connoting sensitivity and sincerity when screening, consider the ethical implications of documenting social needs in health records, and subsequent impact on patient willingness to have needs addressed.

\section{Abbreviations}

$211=$ United Way's 2-1-1, https://uw.org/

$\mathrm{ED}=$ Emergency department

EHR = electronic medical record

REDCap $=$ Research Electronic Data Capture

$\mathrm{SDOH}=$ Social determinants of health

TRA = Theory of Reasoned Action

\section{Declarations}


1. Ethics approval and consent to participate:

a. Human subjects approval and consent: University of Utah Institutional Review Board, Protocol \#0012587

b. Animal study clause: Not applicable

2. Consent for publication: Not Applicable

3. Availability of data and materials: The datasets used and/or analyzed during the current study are available from the corresponding author on reasonable request.

4. Competing interests: The authors declare that they have no competing interests.

5. Funding: This study was supported by the Agency for Healthcare Research and Quality R21 HS026505 (PI: Wallace). Additional support was provided by the National Center for Advancing Translational Sciences of the National Institutes of Health under Award Number The content is solely the responsibility of the authors and does not necessarily represent the official views of the Agency for Healthcare Research and Quality or the National Institutes of Health

6. Authors' contributions: ASW conceived the study design and implementation. BW and JW analyzed and interpreted data regarding staff usage and related informatics. ASW, SMS, and BL were major contributors in writing the manuscript. All authors read and approved the final manuscript.

7. Acknowledgements: We would like to thank Ching-Yu Wang, Ernest Grigorian, Telesia Lelea, Melissa Vega, Patricia Garcia, Caitlin Schneider, Beth Martial, Anna Fonnesbeck, Brieanne Witte, Naomi Flake,and greater UHealth ED Registration team and United Way 211 team for their help with the study's design and implementation.

\section{References}

American Academy of Family Physicians (2020). Assessment and action: Address your patients' social determinants of health. Retrieved on January 25, 2021 at https://www.aafp.org/family-physician/patientcare/the-everyone-project/toolkit/assessment.html

Billioux, A., Verlander, K., Anthony, S., \& Alley, D. (2017). Standardized screening for health-related social needs in clinical settings: The Accountable Health Communities screening tool. NAM Perspectives. https://doi.org/10.31478/201705b

Braveman, P., Egerter, S., \& Williams, D. R. (2011). The Social Determinants of Health: Coming of Age. Annual Review of Public Health, 32(1), 381-398. https://doi.org/10.1146/annurev-publhealth-031210101218

Centers for Disease Control and Prevention (2018). Social determinants of health: Know what affects health. Retrieved from https://www.cdc.gov/socialdeterminants/index.htm

Creswell, J. W. \& V.L. Plano Clark, V. L. (2011). Designing and Conducting Mixed Methods Research. Thousand Oaks, CA: Sage. 
Deci, E. L., \& Ryan, R. M. (1985). Intrinsic motivation and self-determination in human behavior. New York, NY: Plenum.

Finkelstein, A., Zhou, A., Taubman, S., \& Doyle, J. (2020). Health care hotspotting - a randomized, controlled trial. New England Journal of Medicine, 382, 152-162.

https://doi.org/10.1056/NEJMsa1906848

Garg, A., Boynton-Jarrett, R., \& Dworkin, P. H. (2016). Avoiding the Unintended Consequences of Screening for Social Determinants of Health. JAMA, 316(8), 813-814. https://doi.org/10.1001/jama.2016.9282

Glasgow, R. E. (2013). What does it mean to be pragmatic? Pragmatic methods, measures, and models to facilitate research translation. Health Education \& Behavior, 40(3), 257-265.

https://doi.org/10.1177/1090198113486805

Gottlieb, L., Garcia, K., Wing, H., \& Manchanda, R. (2016). Clinical Interventions Addressing Nonmedical Health Determinants in Medicaid Managed Care. American Journal of Managed Care, 22(5), 370.

Gottlieb, L., Tobey, R., Cantor, J., Hessler, D., \& Adler, N. E. (2016). Integrating social and medical data to improve population health: Opportunities and barriers. Health Affairs, 35(11).

https://doi.org/10.1377/hlthaff.2016.0723

Harris, P. A., Taylor, R., Minor, B. L., Elliott, V., Fernandez, M., O'Neal, L., . . Consortium, R. E. (2019). The REDCap consortium: Building an international community of software platform partners. Journal of Biomedical Informatics, 95, 103208. https://doi.org/10.1016/j.jbi.2019.103208

Harris, P. A., Taylor, R., Thielke, R., Payne, J., Gonzalez, N., \& Conde, J. G. (2009). Research electronic data capture (REDCap)-a metadata-driven methodology and workflow process for providing translational research informatics support. Journal of Biomedical Informatics, 42(2), 377-381.

https://doi.org/10.1016/j.jbi.2008.08.010

Heller, C. G., Parsons, A. S., Chambers, E. C., Fiori, K. P., \& Rehm, C. D. (2020). Social Risks Among Primary Care Patients in a Large Urban Health System. American Journal of Preventive Medicine, 58(4), 514-525. doi:https://doi.org/10.1016/j.amepre.2019.11.011

Hsu, C., Cruz, S., Placzek, H., Chapdelaine, M., Levin, S., Gutierrez, F., .. Cheadle, A. (2019). Patient Perspectives on Addressing Social Needs in Primary Care Using a Screening and Resource Referral Intervention. Journal of General Internal Medicine, 35(2). https://doi.org/10.1007/s11606-019-05397-6.

Hwang, S. (2008). Utilizing qualitative data analysis software: A review of Atlas.ti. Social Science Computer Review, 26(4), 519-527. https://doi.org/10.1177/0894439307312485.

LaForge, K., Gold, R., Cottrell, E., Bunce, A. E., Proser, M., Hollombe, C., Dambrun, K., Cohen, D. J., \& Clark, K. D. (2018). How 6 Organizations Developed Tools and Processes for Social Determinants of Health 
Screening in Primary Care: An Overview. The Journal of Ambulatory Care Management, 41(1), 2-14. https://doi.org/10.1097/JAC.0000000000000221

Lewis, C. C., Mettert, K. D., Dorsey, C. N., Martinez, R. G., Weiner, B. J., Nolen, E., Stanick, C., Halko, H., \& Powell, B. J. (2018). An updated protocol for a systematic review of implementation-related measures. Syst Rev, 7(1), 66. https://doi.org/10.1186/s13643-018-0728-3.

Kim, H., Sefcik, J. S., \& Bradway, C. (2017). Characteristics of qualitative descriptive studies: A systematic review. Res Nurs Health, 40(1), 23-42. https://doi.org/10.1002/nur.21768.

Klesges, L. M., Estabrooks, P. A., Dzewaltowski, D. A., Bull, S. S. \& Glasgow, R. E. (2005). Beginning with the application in mind: Designing and planning health behavior change interventions to enhance dissemination. Annals of Behavioral Medicine, 29(2), 66-75.

Sandelowski M. (2000). Whatever happened to qualitative description? Res Nurs Health, 23(4), 334-40. https://doi.org/10.1002/1098-240x.

Stanick, C. F., Halko, H. M., Dorsey, C. N., Weiner, B. J., Powell, B. J., Palinkas, L. A., \& Lewis, C. C. (2018). Operationalizing the 'pragmatic' measures construct using a stakeholder feedback and a multi-method approach. BMC Health Services Research, 18(1), 882. https://doi.org/10.1186/s12913-018-3709-2.

Sullivan H. R. (2019). Hospitals' obligations to address social determinants of health. AMA Journal of Ethics, 21(3), E248-E258. https://doi.org/10.1001/amajethics.2019.248

Tong, S. T., Liaw, W. R., Kashiri, P. L., Pecsok, J., Rozman, J., Bazemore, A. W., \& Krist, A. H. (2018). Clinician experiences with screening for social needs in primary care. Journal of the American Board of Family Medicine, 31(3), 351-363. https://doi.org/10.3122/jabfm.2018.03.170419

Tsai, J., Gelberg, L. \& Rosenheck, R.A. (2019). Changes in physical health after supported housing: Results from the Collaborative Initiative to End Chronic Homelessness. Journal of General Internal Medicine, 34, 1703-1708. https://doi.org/10.1007/s11606-019-05070-y.

Venkatesh, A. K., Chou, S. C., Li, S. X., Choi, J., Ross, J. S., D'Onofrio, G., Krumholz, H. M., \& Dharmarajan, K. (2019). Association Between Insurance Status and Access to Hospital Care in Emergency Department Disposition. JAMA Internal Medicine, 179(5), 686-693.

https://doi.org/10.1001/jamainternmed.2019.0037

Wallace, A. S., Luther, B., Guo, J. W., Wang, C. Y., Sisler, S., \& Wong, B. (2020). Implementing a Social Determinants Screening and Referral Infrastructure During Routine Emergency Department Visits, Utah, 2017-2018. Prev Chronic Dis, 17, E45. https://doi.org/10.5888/pcd17.190339

Walker, R.J. \& Jackson, J.L. (2019). How Do We Address the Influence of Social Determinants on Health?. Journal of General Internal Medicine, 34, 1673-1674. https://doi.org/10.1007/s11606-01905167-4. 
Zhou, R. A., Baicker, K., Taubman, S., \& Finkelstein, A. N. (2017). The uninsured do not use the Emergency Department more-they use other care less. Health Affairs, 36(12), 2115-2122. https://doi.org/10.1377/hlthaff.2017.0218.

\section{Figures}

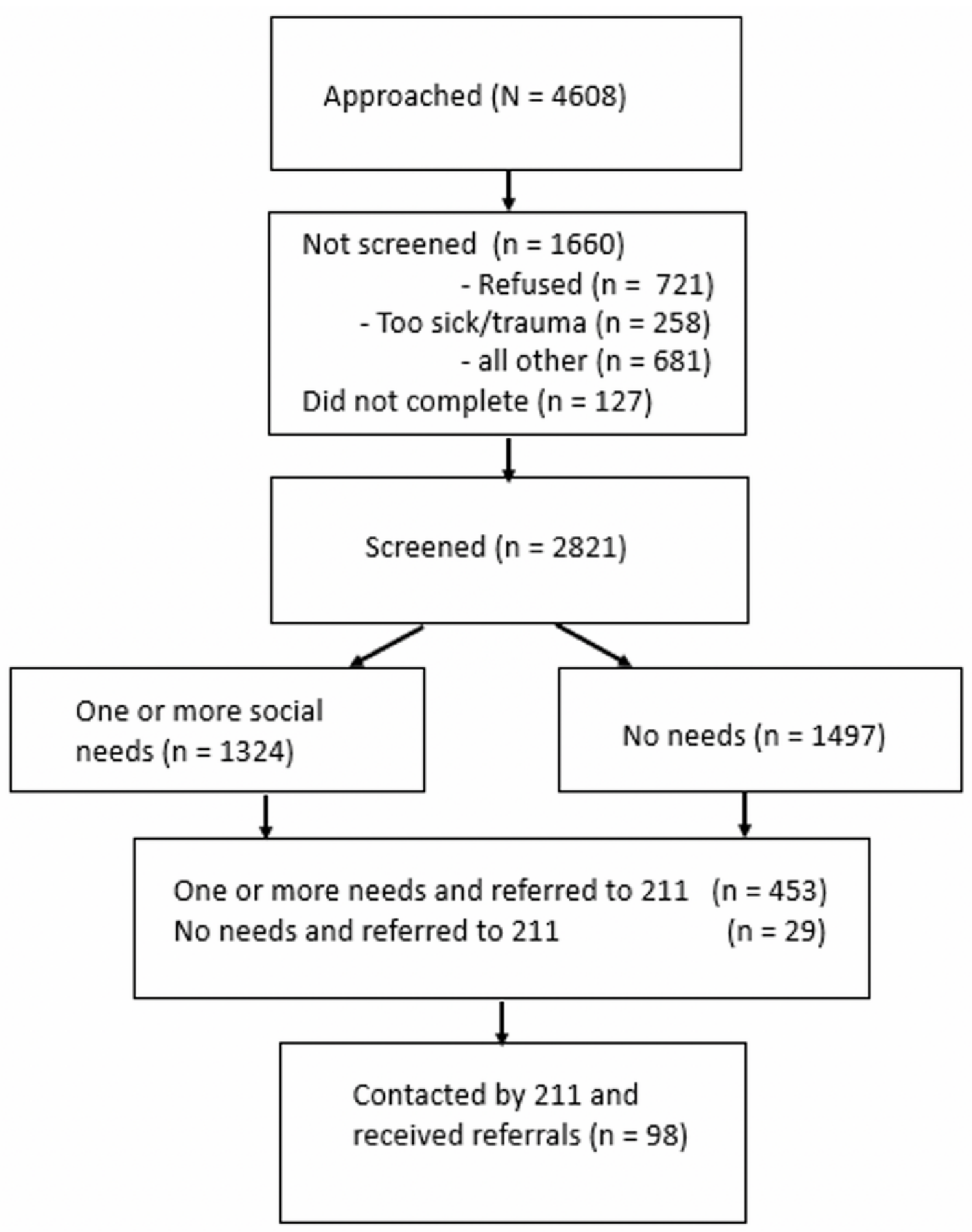

Figure 1 
Retention of Patients from Approach to Service Referrals

\section{Supplementary Files}

This is a list of supplementary files associated with this preprint. Click to download.

- ImplSciCommStaRIchecklistforauthorcompletion.docx

- ImplSciCommVoltageDropAppendixA.docx

- ImplSciCommVoltageDropAppendixB.docx

- ImplSciCommVoltageDropAppendixC.docx 